\title{
Nurses' workarounds in acute healthcare settings: a scoping review
}

Deborah S Debono ${ }^{1 *}$, David Greenfield ${ }^{1}$, Joanne F Travaglia ${ }^{1,2}$, Janet C Long ${ }^{1}$, Deborah Black ${ }^{3}$, Julie Johnson ${ }^{1}$ and Jeffrey Braithwaite ${ }^{1}$

\begin{abstract}
Background: Workarounds circumvent or temporarily 'fix' perceived workflow hindrances to meet a goal or to achieve it more readily. Behaviours fitting the definition of workarounds often include violations, deviations, problem solving, improvisations, procedural failures and shortcuts. Clinicians implement workarounds in response to the complexity of delivering patient care. One imperative to understand workarounds lies in their influence on patient safety. This paper assesses the peer reviewed empirical evidence available on the use, proliferation, conceptualisation, rationalisation and perceived impact of nurses' use of workarounds in acute care settings.

Methods: A literature assessment was undertaken in 2011-2012. Snowballing technique, reference tracking, and a systematic search of twelve academic databases were conducted to identify peer reviewed published studies in acute care settings examining nurses' workarounds. Selection criteria were applied across three phases. 58 studies were included in the final analysis and synthesis. Using an analytic frame, these studies were interrogated for: workarounds implemented in acute care settings by nurses; factors contributing to the development and proliferation of workarounds; the perceived impact of workarounds; and empirical evidence of nurses' conceptualisation and rationalisation of workarounds.
\end{abstract}

Results: The majority of studies examining nurses' workarounds have been published since 2008, predominantly in the United States. Studies conducted across a variety of acute care settings use diverse data collection methods. Nurses' workarounds, primarily perceived negatively, are both individually and collectively enacted. Organisational, work process, patient-related, individual, social and professional factors contribute to the proliferation of workarounds. Group norms, local and organisational culture, 'being competent', and collegiality influence the implementation of workarounds.

Conclusion: Workarounds enable, yet potentially compromise, the execution of patient care. In some contexts such improvisations may be deemed necessary to the successful implementation of quality care, in others they are counterproductive. Workarounds have individual and cooperative characteristics. Few studies examine nurses' individual and collective conceptualisation and rationalisation of workarounds or measure their impact. The importance of displaying competency (image management), collegiality and organisational and cultural norms play a role in nurses' use of workarounds.

Keywords: Workaround, Violation, Deviation, Short cut, First order problem solving, Patient safety, Procedural failure

\footnotetext{
* Correspondence: d.debono@unsw.edu.au

${ }^{1}$ Centre for Clinical Governance Research, Australian Institute of Health Innovation, University of New South Wales, Sydney, NSW 2052, Australia Full list of author information is available at the end of the article
} 


\section{Background}

Workaround behaviours are those that circumvent or temporarily 'fix' an evident or perceived workflow block. Workplace workarounds are used to: solve problems [1,2]; sidestep 'problematic' rules [3]; bypass workflow blocks created by safety mechanisms [4]; address poor workflow design [5] and organisational and system issues [3]; save time [6]; backup software data applications [7]; compensate for inadequate technology [8,9]; patch software glitches [10]; or offer solutions to a range of problems including shortcomings in staffing, equipment and supplies [11]. Workarounds are claimed to increase when the complexity of the task is incompatible with the degree of structure imposed by the system $[12,13]$ and when users feel 'controlled' by the system [14], with end user resistance contributing to their implementation [15].

Views about workarounds tend to polarise. On the one hand, negative conceptualisations report workarounds as a subset of errors, shortcuts, deviations and violations $[3,16]$. Terms such as improvisations $[10,17]$ and innovations [18] offer more positive notions of workarounds. There is a paucity of clear and uniform definitions of these related constructs $[3,16]$. The definition of workarounds developed for this review has two components. Workarounds are observed or described behaviours that may differ from organisationally prescribed or intended procedures. They circumvent or temporarily 'fix' an evident or perceived workflow hindrance in order to meet a goal or to achieve it more readily.

Healthcare is a high-hazard industry in which workers have the potential to kill or maim [19:85]. More than most other industries, healthcare is complex, fragmented, decentralised and unevenly regulated [19] with clinicians required to learn on the job at the same time as they are required to display professional autonomy. Healthcare is characterised simultaneously by routine, highly organised and ultra safe practices (e.g. blood product protocols) and unpredictable, erratic hazardous demand. It is comprised of both long-term patient-clinician relationships (e.g. chronic disease) and acute, fleeting interactions (e.g. outpatient and emergency department episodes) [20]. These features of healthcare shape the way people work, behave and respond to the demands of clinical practice. Rules, policies and technologies seek to standardise clinicians' practice. Clinicians seem to implement workarounds as a way of responding to the complexity of care within a system that increasingly demands standardisation. Although nurses are touted the masters of workarounds [21] the empirical literature focusing on them has been slow to flourish [16]. Nurses comprise the majority of the healthcare workforce. Therefore while acknowledging the corpus of literature on workarounds in other industries [22], this study focuses on nurses' behavioural workarounds in acute healthcare organisations.
One imperative to understand workarounds in healthcare lies in their influence on safe care. Workarounds can both subvert and augment patient safety. In circumventing safety blocks [4], masking deficiencies [23,24], and undermining standardisation [25], they potentially jeopardise care to patients. Conversely, workarounds operate as localised acts of resilience $[26,27]$, are at times crucial to the delivery of services [4], operate as adaptions to inefficiencies [20] and provide opportunities for improvement [28].

To enervate the negative and harness the positive potential of workarounds in healthcare, we must firstly understand the factors that influence their implementation and proliferation and the role of local and organisational culture in shaping them. This premise underpins this review, the purpose of which is: to assess the peer reviewed empirical evidence available on the use, proliferation and perceived impact of workarounds by nurses in acute care settings; and to examine how they are conceptualised and rationalised by those who use them.

Given the significance of the topic and the increase in publications in this area since 2008, it is timely to review the literature on workarounds. A 2008 review of workarounds in healthcare settings concluded that because there are so few studies that have empirically studied work-arounds "it was not possible to produce a typical quantitative review of the literature" [16:3]. A 2009 review of the empirical literature examining a construct overlapping with workarounds, rule violations in work settings, identified that this too is an area requiring further work [3]. Since the publication of these literature reviews significantly more work has been published in this area (Table 1).

\section{Method \\ Scope}

While our paper narrows the focus of Halbesleben et al's 2008 review [16] to workaround behaviours of nurses, it also broadens the enquiry to examine literature from a wider range of disciplines including Safety Science and Sociology. It also includes a greater variety of search terms to capture empirical literature on behavioural workarounds used by nurses. We differentiate from the work by Alper and Karsh [3], by narrowing the focus to nurses' behavioural workarounds (including situational violations). Our study builds on both reviews by also

Table 1 Scopus search using search term (workaround* OR work-around) [accessed $5^{\text {th }}$ March 2012]

\begin{tabular}{ll}
\hline Year & Number of references identified in Scopus \\
\hline $2008-2012$ (<4 years) & 517 \\
$2000-2007$ (7 years) & 429 \\
$1961-1999$ (38 years) & 251 \\
\hline
\end{tabular}


examining nurses' collective and individual conceptualisation and rationalisation of workarounds. These criteria allow for a more detailed and nuanced examination of workarounds.

Scoping methodology offers an opportunity to develop an understanding of multiple perspectives on a single issue [29]. We adopted a similar approach used in other studies [30] and diverged from the methodology described by Levac and colleagues [31] by excluding the final step of a six step framework, stakeholder consultation, which was not relevant for this study. We conducted a scoping review for several reasons. Workarounds are not yet a clearly indexed concept in academic literature databases. A systematic review involves a clearly defined topic and question. The examination of workarounds and safety violations is a pluralistic and expanding area informed by methodologically diverse research. The findings of these disparate methods do not easily lend themselves to traditional systematic reviews and meta-analyses [32]. The scoping method involves review, analysis and synthesis of a broad scope of literature. Unlike systematic reviews, scoping studies do not assess the quality of studies [31] and as they require the literature to be analytically reinterpreted they differ from narrative literature reviews as well [31]. This method is appropriate given the complexity of the area and the aim, which is to build a comprehensive picture of workarounds, rather than to weigh up the levels of evidence in relation to a specific question. The process is outlined in Figure 1.

\section{Search strategy}

A multi-method search strategy was employed. The snowball method and reference tracking were used in conjunction with a systematic search of academic databases. The snowball method included checking references of relevant papers, serendipitously identified references, alerts and citation tracking. Studies identified in this manner up until 30th May 2012 were included for analysis.

Academic literature databases, initial search terms, limiters, inclusion and exclusion criteria were determined a

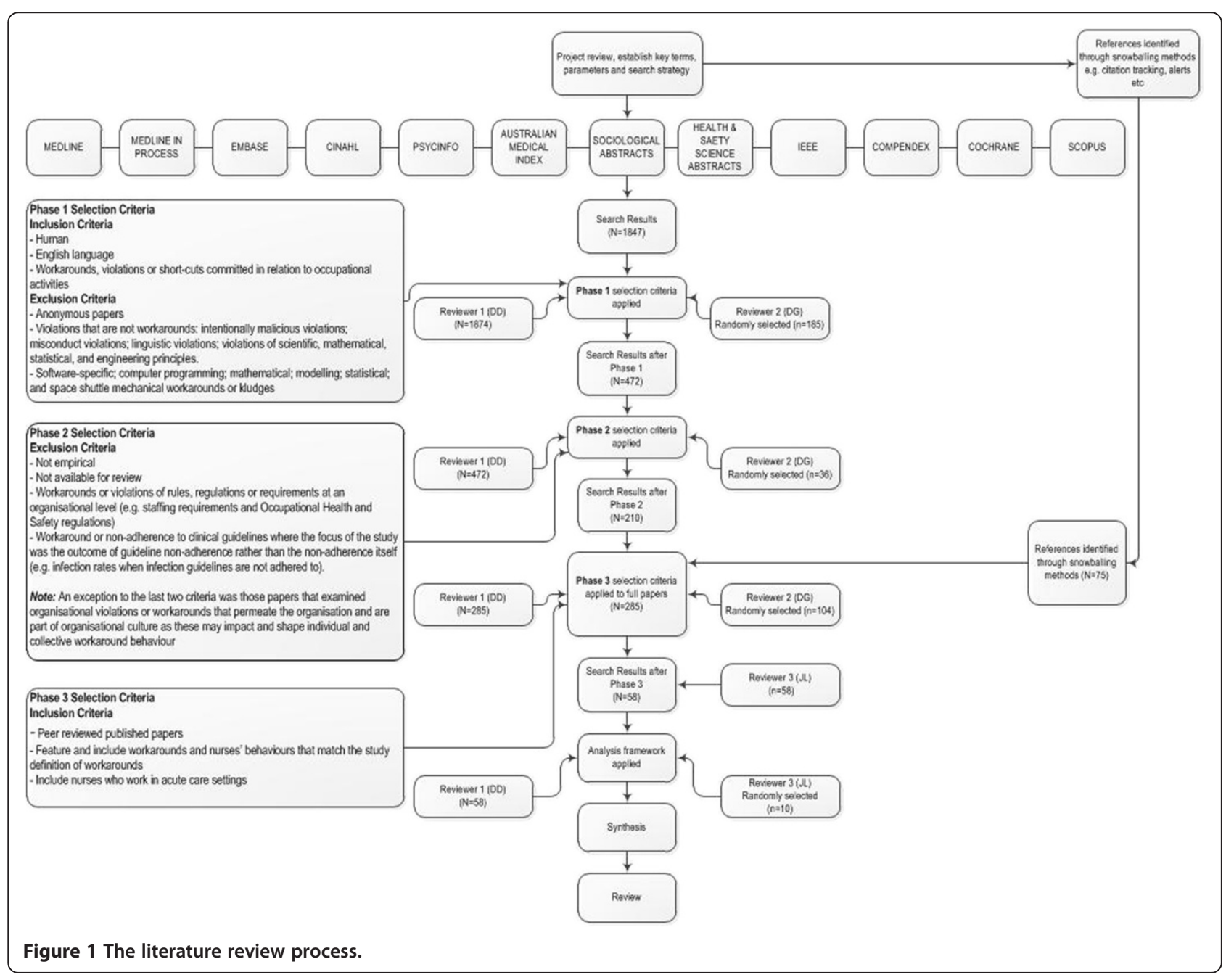


priori utilising brainstorming and mind mapping techniques. An iterative process was then employed involving a preliminary review of key references and discussion with experts in literature searching techniques to hone search strategies and terms. References in articles that met the selection criteria were then searched as a way of identifying seminal articles and then tracking papers that had cited these references [3]. Key words, controlled and uncontrolled index terms in relevant references identified discipline-specific search terms were used. Consultation with a specialist university research librarian in August 2011 confirmed the search strategy and provided expertise and advice. The academic literature databases searched included: Medline; Medline in Process; Embase; Cinahl; PsycInfo; Australian Medical Index; Sociological Abstracts; Health and Safety Science; IEEE; Compendex; Cochrane Database of Systematic Review; and Scopus. All databases were interrogated using the search terms workaround*/ work-around"/'work around'; and Violation* + Safety + Rule*/Policy. In addition, Medline; Medline in Process; Embase; Cinahl; PsycInfo; Australian Medical Index; Sociological Abstracts; Health and Safety Science data bases were searched using the search terms: short-cut*/shortcut*; violation"; problem-solving; 'temporary fix*; 'informal practice*'; 'informal interaction "'; 'creative solution*'; deviation*'; and 'procedural error"' cross-tabulated with nurs".

Search terms were subjected to standardised procedures. Truncation of the search term allowed for the search of plurals and other suffixes. Enclosing the search term within quotation marks restricted the search to the exact phrase. Limiters "human" and "English language", "NOT prison OR parole" were used when available.

Following the removal of duplicate and non-English references 1847 references remained. References were examined against the selection criteria as outlined following.

\section{Selection criteria}

Selection criteria were developed both a priori and through an iterative process that involved examination of the references and discussion between two authors (DD and DG) across three phases. At each phase, the selection criteria were refined to capture only those studies relevant to the review objective (Figure 1). Post hoc development of selection criteria is an integral part of the scoping review process [30]. In Phase 1 the selection criteria were broad to include papers examining workarounds, violations or short-cuts committed in relation to occupational activities. Additionally, the selection criteria were designed to screen out papers examining violations that are not workarounds: intentionally malicious violations (e.g. physical, sexual and human rights violations); misconduct violations (e.g. sporting, contract, copyright, privacy and parole violations); linguistic violations; violations of scientific, mathematical, statistical and engineering principles.
We excluded papers examining: software-specific; computer programming; mathematical; modelling; statistical; and space shuttle mechanical workarounds or kludges. We also excluded papers at this phase that were not written in English.

The purpose of Phase 2 was to further exclude papers if they met additional screening criteria. That is, papers that examined: workarounds or violations of rules, regulations or requirements at an organisational level (e.g. staffing requirements and occupational health and safety regulations); and workarounds or non-adherence to clinical guidelines where the focus of the study was the outcome of guideline non-adherence rather than the non-adherence itself (e.g. infection rates when infection guidelines are not adhered to). An exception to these criteria was those papers that examined organisational violations or workarounds that permeate the organisation and are part of organisational culture. These were included as they may impact and shape individual and collective workaround behaviour.

Following application of selection criteria in Phases 1 and 2, there were 210 references identified through academic database searches remaining. In addition, 75 references had been identified as relevant at face value via snowballing. The Phase 3 selection criteria were applied to these 285 references. Full papers were scrutinised and included if they met the following criteria: peer reviewed published papers; featured and included workarounds and nurses' behaviours that matched our definition of workarounds; and involved nurses who worked in acute care settings. We adopted a conservative approach, including rather than excluding studies. There remained 44 papers identified through academic database searching and 14 identified through the snowball technique that were eligible for inclusion in the review. Two authors (DD and JL) independently examined the 58 remaining papers against the selection criteria and were in agreement regarding their inclusion.

\section{Analysis and synthesis}

An analytic frame reflecting the objective of the study was developed by two of the authors (DD and DG) (see below). The first author (DD) used the analytic frame to interrogate all of the papers that met the selection criteria $(\mathrm{N}=58)$. Using a random number generator, 10 of the included studies were selected. A second author (JL) interrogated these 10 studies (17\%) independently using the analysis framework. The two authors (DD and JL) compared their findings. The authors were in agreement on the extracted data. The findings are organised into categories based on the analysis framework [33]: workarounds implemented in acute care settings by nurses; factors contributing to the development and proliferation of workarounds; the perceived impact of 
workarounds; and empirical evidence of nurses' conceptualisation and rationalisation of workarounds.

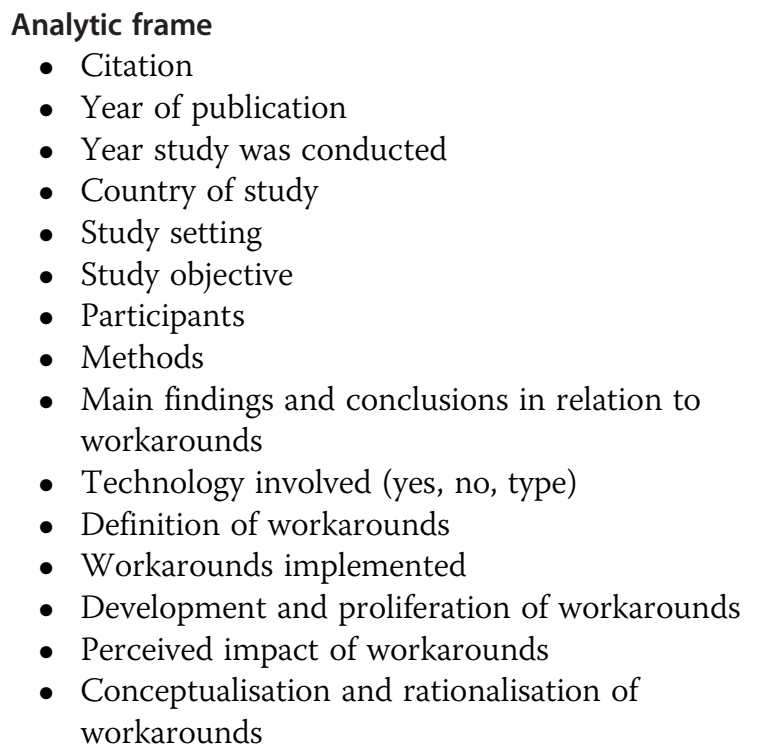

\section{Results}

Key study features

Just over half of the studies reviewed (59\%) were published between 2008-2012, with the mode being 2009 $(n=9)$. Empirical evidence on workarounds arises predominantly from studies conducted in acute care settings in the United States of America (USA) $(n=29)$. The United Kingdom (UK) $(\mathrm{n}=8)$ [34-41], Australia $(\mathrm{n}=5)$ [42-46], The Netherlands $(n=5)$ [47-51], Canada $(n=3)$ [52-54], Japan $(\mathrm{n}=1)$ [55], Lebanon $(\mathrm{n}=1)$ [56] and Thailand ( $\mathrm{n}=1)$ [57] also hosted studies examining workarounds. Additionally, four studies were conducted in both Canada and the USA $[1,24,58,59]$ (Table 2).

Study settings comprised hospitals that provided general medical, specialised paediatric and psychiatric services [60], and a variety of wards including, but not limited to: intensive care $[1,4,35,58,59,61-66]$, medical and surgical $[1,4,35,45,46,58,59,62,64,66-70]$, oncology $[1,50,58,59,62,64,65]$, maternity $[1,58,59,62,66,67,71]$, cardiac units $[1,45,50,58,59]$, operating theatre units $[38,52,62,72]$, emergency and trauma departments $[35,57,58,62,73-75]$, outpatient clinics [76] and paediatrics $[1,35,40,53,54,58-60,63-66,71]$. These wards were identified in academic $[48,49,58,64,71,75,77,78]$ and nonacademic [58,61,72], community $[1,58,59,61,62,72,79]$, tertiary $[45,58,64]$ and teaching hospitals $[1,40,46,52-$ $54,59,79]$, in urban $[1,45,53,58,59,64,69]$ and rural $[1,43,44,58,59,61]$ settings (Table 2). Our examination focused on nurses' workarounds but a number of studies also incorporated other professional groups including doctors $[4,34,37,39,48-50,53,54,56,72-76,80-82]$,

Table 2 Country and setting in reviewed studies

\begin{tabular}{|c|c|c|c|}
\hline \multicolumn{2}{|l|}{ Country of study } & \multicolumn{2}{|l|}{ Study setting } \\
\hline United States of America & $\begin{array}{l}{\left[4,6,60,61,62^{*}, 63,64,65^{*}, 67,68,\right.} \\
69^{*}, 70-73,74^{*}, 75,76,77^{*}, 78-81 \\
\left.82^{*}, 83-87\right]\end{array}$ & Intensive Care Units & {$[4,35,40,58,59,61-66]$} \\
\hline Not specified & [66] & Medical and surgical Units & {$[1,4,34,35,40,45,46,48,58,59,62,64,66-70]$} \\
\hline United Kingdom & [34-41] & Oncology Units & {$[1,34,41,50,58,59,62,64,65,84]$} \\
\hline Australia & {$[42-46]$} & Maternity Units & {$[1,58,59,62,66,67,71]$} \\
\hline The Netherlands & {$[47-51]$} & Cardiovascular Units & {$[1,34,40,45,50,53,58,59,66]$} \\
\hline Canada & {$[52-54]$} & Operating Theatre & {$[38,52,62,72,77]$} \\
\hline Canada and United States & {$\left[1,24^{*}, 58,59^{*}\right]$} & Emergency and trauma Units & {$[35,57,58,62,73-75]$} \\
\hline Japan & {$[55]$} & Psychiatry Units & [66] \\
\hline Lebanon & {$\left[56^{*}\right]$} & Long term care Units & {$[6,84]$} \\
\hline \multirow[t]{8}{*}{ Thailand } & {$[57]$} & Neurology Units & {$[46,51,78]$} \\
\hline & & Pediatrics & {$[35,40,53,54,58,59,63-66,71,77]$} \\
\hline & & Other & {$[40,46,51]$} \\
\hline & & Veteran Affairs Medical Centers/Hospitals & {$[6,76,80,81,84]$} \\
\hline & & Community Hospitals & {$[1,58,59,62,72,79]$} \\
\hline & & Tertiary Hospitals & {$[58,64,67]$} \\
\hline & & Teaching/University/Academic Hospitals & $\begin{array}{l}{[1,34,46,48,49,52-} \\
54,56,58,59,64,67 \\
68,71,75,77-79,82,84]\end{array}$ \\
\hline & & Non Academic/Non Teaching Hospitals & {$[34,40,58,72]$} \\
\hline
\end{tabular}

${ }^{*}$ Authors contacted. 
pharmacists $[4,34,35,48,50,56,70,80,81,83,84]$, information technology staff $[4,56,80,81,84]$ and other hospital employees $[60,72,75,78,79,81]$.

While some studies used a single data collection method ( $\mathrm{n}=21$ ), this review has identified that the majority of studies investigating this topic have engaged a multi method approach $(\mathrm{n}=37)$ (Table 3). A combination of interview and observation was the most frequently used multi method combination. An unusual method of data collection recorded nurses' talk about what they were doing and thinking as they were administering medication [67].

The term 'workaround' was defined in less than $30 \%$ $(\mathrm{n}=17)$ of the studies reviewed $[4,34,48-50,54,56,60$, $61,63,67,71,72,74,77,79,82]$. Definitions of workarounds predominantly articulated intent to achieve an outcome through handling failures and exceptions in workflow $[48,72,74]$ or by bypassing formal rules, protocols, standards or procedural codes $[4,49,50,54,56,61,67]$. Negative and positive views of workarounds were evident in the wording of several definitions. Positive aspects of workarounds include benefits for patients [67], increased efficiency for nurses [67] and a way for nurses to avoid harmful or unrealistic expectations [71]. Other definitions of workarounds convey a negative message with workarounds described as non-compliant [56], at risk, unsafe behaviours [79]. Definitions in two of the studies intimate the simultaneous negative and positive characteristic of workarounds $[50,60]$. Of those studies that defined workarounds, almost three quarters $(71 \%)$ were published between 2009-2012.

We included seven studies $[39,40,43,44,55,64,65]$ that offered definitions for violations because the definition incorporated elements common to the definition of a workaround or the described behaviours aligned with the definition of a workaround. For example, violations as necessary deviations such as having to break protocol (authors' emphasis) [64] or shortcuts [43]. Violations were employed as a way of working around rules, regulations, policies, procedures and recommendations. Definitions of violations offered in two of the seven studies specified that violations were not intended to harm [40,43]. In other studies, definitions offered for first order problem solving and deviations matched our definition of workarounds $[24,58,59,73]$. In this paper we use the term workaround to refer to behaviours that

Table 3 Data collection methods in reviewed studies

\begin{tabular}{|c|c|}
\hline Method & Studies \\
\hline \multicolumn{2}{|l|}{ Discrete Method } \\
\hline Observations & {$[62,73]$} \\
\hline Interviews & {$[36,52,80,81]$} \\
\hline Focus group interviews & {$[69,86]$} \\
\hline Questionnaire surveys & {$[37,39,43,44,55,60,63-65,79,83,87]$} \\
\hline Information system data analysis & [77] \\
\hline \multicolumn{2}{|l|}{ Multi-method } \\
\hline Interview and observation & $\begin{array}{l}{\left[1,6^{*}, 34,40,41,45,53,54,56,58,59,\right.} \\
\left.61,66,68,72,74,75,76^{*}, 82,84^{*}\right]\end{array}$ \\
\hline Interview and document analysis including medication chart review & {$[48,51]$} \\
\hline Interview, observation and document analysis (may include medication chart review) & {$[38,42,50]$} \\
\hline Interview, observation, focus group, survey and time and motion studies & [78] \\
\hline Analysis of information system data and observation & [70] \\
\hline Analysis of information system data, observation and interview & [4] \\
\hline Observation, clinical intervention data and medication chart review & [35] \\
\hline Observation and medication chart review & [46] \\
\hline Interview and collection of data from support desk and information system data & [85] \\
\hline Questionnaire surveys, observations, interviews and Computer Provider Order Entry (CPOE) website review & [71] \\
\hline Questionnaire surveys and observation and focus groups & [57] \\
\hline Questionnaire surveys and interviews & [49] \\
\hline Questionnaire surveys, interviews, process mapping, information system data and document analysis & [47] \\
\hline Observation and journal narration & [24] \\
\hline Self-recording by nurses as they gave medication and interviews & [67] \\
\hline
\end{tabular}

*Observational studies that noted inclusion of 'complementary' and 'opportunistic' interviews. 
match the definition in this study (e.g. first order problem solving).

\section{Workarounds implemented by nurses}

Papers were examined for examples of behaviours that matched our operational definition of workarounds. The majority of studies offered exemplars of workarounds $(\mathrm{n}=46)$. However, some did not detail workaround practices $[1,38,43,47,55,60,62-64,73,83]$. While in most studies examples of behaviour were clearly workarounds, there were some studies in which it was more difficult to determine and for these it was necessary to consult the offered causes of the behaviour to determine whether it could be defined as a workaround. For example, we defined the practice of not checking the identification (ID) band as a workaround when a suggested barrier to accomplishing the goal of administering the medication is the time taken to check the ID band [46]. One study examined nurses working around the need to report errors by redefining errors [42].

\section{Workaround categories}

Nurses' workarounds in acute care settings have been studied predominantly in relation to technology including barcode medication administration (BCMA) features $[4,6,34,51,63,65,67,68,70,84,85]$, Computer Provider Order Entry (CPOE) [47-50,71,82], electronic health record (EHR) $[53,54,76,80,81]$, smart pumps for intravenous infusion [77,86], equipment [69], test ordering [75] and pharmacy dispensing [56]. Workaround behaviours have also been examined as a response to: operational failures; time pressures; and perceived workflow restraints [1,24,58,59,61,62,72,74,78]; expectations [44,58,59,72]; and rules (formal and cultural), policies, guidelines and regulations [36-40,42-44,46,52,55-57,64-67,73,83,87]. We grouped these into three categories: technology; operational failures and work restraints; and policies, rules and regulations. Workarounds within these categories fall into two broad groups, those that are enacted individually and those that are enacted collaboratively. Many studies portrayed participant involvement that was both collaborative and individual and described workaround behaviours that fell into more than one category. To illustrate, scanning a patient barcode on a sticker rather than on the patient's armband is an individually enacted workaround in response to technology and policy requirements [4].

The majority of described individually enacted workarounds involve responses to technology and policy particularly in relation to medication administration. Examples of collectively and individually enacted workarounds are provided in Table 4.

\section{Factors contributing to the development and proliferation of workarounds}

We examined the studies for factors identified as leading to the development of workarounds. We also sought evidence for those factors that encourage or enable established workarounds to continue, for example, nurses sharing or teaching workarounds to junior staff $[53,69]$.

Workarounds develop in response to factors that are perceived to prevent or undermine nurses' care for their patients or are not considered in the best interest of their patients, make performance of their job difficult, or potentially threaten professional relationships. These factors can be categorised as organisational, work process, patient, individual clinician and relational/professional factors.

\section{Organisational factors}

Staffing levels, the need to manage heavy and fluctuating workloads (working in crisis mode) [78] and productivity pressures were commonly offered organisational causes of workaround behaviours [4,24,42-44,51,52,58,59,62, $78,83,87]$. In addition negative organisational climate characterised by poor leadership, a lack of involvement of nurses in decision-making, few opportunities for professional development and a lack of perceived human management resources and support contributed to the development of workaround behaviours $[4,43,58,60,66]$. Other factors include organisational expectations that clinicians multitask [52], a lack of role clarity [4,52], ambiguity [62], organisational processes that have not been re-engineered to fit with the implementation of technology [48,71], the low status of nurses [24] and organisational guidelines and group norms that prevent visible and formal expression of emotion about patients [75].

\section{Work process factors}

An array of work process factors giving rise to workaround behaviours were identified in the studies reviewed. The mismatch between introduced technology or policies and current workflow was one of the most common causes of workaround behaviours [4,6,34,42, 47-51,53,54,56,61,63,68-71,76-78,80-82,84-86]. Operational failures including resource issues, equipment not stocked properly, documentation not completed, missing information and medications and environmental factors [1,4,35,44,57-59,61,69,72,78,85] were also typical precursors to workaround behaviours. Similarly, heavy workloads, time constraints or attempts to increase efficiency led to workaround behaviours $[4,6,24,45,49,51,57-59,67$, 69,76,80-84]. Workaround behaviours were also attributed to the complexity and dynamic conditions of clinical work $[72,74,80,81]$, including interruptions $[61,68$, $83,84]$, emergencies $[44,50,52,57,61,64,67,71]$ and the lack of availability of doctors to provide information $[44,48,61,66]$. Studies identified that in situations there 
Table 4 Illustrative examples of workarounds

\section{Factors \\ Studies that provided \\ examples of individually \\ enacted workarounds \\ Illustrative examples of individually \\ exacted workarounds}

$[4,6,34,48,49,51,53,54,63$,

Technology, Characteristics of the technology that impose workflow blocks/delays

- In a study examining nurses use of BCMA nurses were observed to "batch" and prepour medications which involves scanning medications and multiple ID bands for multiple patients before commencing medication administration [6]

- In a study examining the use of a CPOE system, dead zones caused the computers to freeze so the nurses used paper lists of pertinent patient information, surgery lists, whiteboards, and other computers to enhance communication and ensure that timely care was given [71]

- In a study examining the side effects of BCMA introduction, nurses were observed to workaround scanning wristbands on patients by typing in the 7-digit number because it took less time than wheeling the medication cart into the patient's room, the patient was isolated, did not have a band on, or the wristband barcode did not scan reliably [84]

\section{Operational failures, exceptions and work} restraints, Issues that make it difficult to

complete the task: resource and equipment $[24,35,36,40,44,48,49,57$ $59,61,65,66,69,76,78,80$ $81,84,85]$

information; knowledge; others' actions
- A study examining the universal precaution practices of nurses in an ED, offers several examples of workarounds including nurses resheathing needles to workaround the distance to the disposal container and to facilitate dislodging needles from syringes; not wearing gloves to workaround the perceived greater risk of needle stick injury if the gloves were the wrong size [57]

- In examining the relationship between work constraints imposed on nurses and patient falls, nurses were identified to multi task, keeping mental track of where they are up to in their list of tasks (cognitive head data). To work around the constraints of too much cognitive head data, nurses use written and mental chunking schemas (e.g. visual reminders and chunking groups of tasks) [78]

\section{Studies that}

provided examples

of collaboratively

enacted workarounds

$[4,6,48-50,53,54,56$

$68,69,71,76,80-82]$

- A study examining use of a CPRS identified a paper-based workaround in which doctors write orders on paper and get the nurses to input them in the CPRS and the doctor signs the nurse-entered orders later [80]

- There were several workarounds described in a study that compared a paper-based and electronic prescribing system. For example, in the CPOE there was a similarity between the Start and Stop orders which nurses worked around by using a STOP stamp on the paper chart to indicate that the medication should be stopped. Another workaround involved nurses writing new times for administration on the paper Kardex but not entering these new times in the CPOE because nurses were blocked from making changes to orders in the system [50]

\section{$[24,42,48,49,59,61,67$, $69,72,74,76,78,80,81]$}

-

- A study examining rework and workarounds in hospital medication administration processes reported that when nurses were unable to understand a medication order they worked around this barrier by asking other nurses', clerks', pharmacists' opinions or make a decision without calling the physician because they did not want to bother or feared repercussions from bothering the physician [61]

- A study of the relationship between nurses work constraints and patient falls identified that nurses workaround the constraints imposed by a lack of formal handover between registered nurses and assistant nurses by informal querying of the previous care nurse about fall status and use of visual cues e.g. stickers [78] 
Table 4 Illustrative examples of workarounds (Continued)

\section{Rules/policies/guidelines/regulations, Formal [4,34-36,40,41,44-}

rules, policies, guidelines, regulations regarding $\quad 46,48,49,57,61,65,66,68$

delivery of care
$71,79,82,84,85,87]$
- A study assessing the impact of a CPOE system noted that when physicians had not yet entered medication orders in the system, nurses worked around the delay by

beginning medication work based on the notes they took during medical rounds [49]

- A study examining baby feeding practices by midwives in 2 UK hospitals, identified that while feeding breast fed babies a bottle of artificial milk was not evidence-based practice and against policy, midwives secretly gave bottles of artificial milk at night, working around espoused policy requirements by

calling it a 'special' cup feed (a cup feed

being acceptable to policy) [36]
- The clinicians work around the policy that requires completion of an authorisation form for a restricted antibiotic to be dispensed [56]
- Collaboration is needed to work around error reporting by redefining the error. For example, a nurse may be given the medication chart from the day before to fix because she/he forgot to record it on their last shift [42]
$[4,6,42,48-50,52,56,67$ $68,71,75,80,81,84]$


were conflicting goals [84], or where nurses perceived particular requirements as less important, appropriate, useful or necessary, they were more likely to work around them $[6,36,45,57,65,87]$. To illustrate, in studies comparing medication administration workarounds across wards, not checking patient identification [64] and scanning a 'surrogate' wristband, which is not on a patient [6] were found to be more common in long-term care wards suggesting that the imperative to check patient identification was less because the nurses were familiar with the patients $[6,64]$.

\section{Patient related factors}

One of the most frequently identified motives for implementing workarounds was the need to ensure that patients received care in a timely manner $[4,44,45,49,61$, $67,82,84,85]$. Other justifications included a perception that rules and policies are not always in the best interest of the patient $[36,42,66]$, the importance of customising care to the need of patients $[4,6,42,72,84]$, patient isolation $[4,68,84]$ and unavailability $[6,34]$ and concern about the impact of adhering to policy on patients' perceptions (e.g. wearing gowns, gloves and masks [57] and repeatedly checking patient identification [41]).

\section{Individual clinician factors}

Causes of workarounds located with the individual were presented by some studies. These included fatigue $[79,83]$, cognitive load $[48,78,80,81]$, unfamiliarity with the technology or its safety features, or a perception that they are not critical or efficient $[4,80,81]$. In some cases nurses unknowingly use workarounds when they are unaware of hospital policies [4]. Nurses are more likely to work around rules if they do not know the content or meaning of the rule or policy [45,55], they believe they are unnecessary [57], they do not approve of them [36] or if following a rule was perceived to carry more risk than not [57].

Workarounds in relation to a new electronic system were attributed to individual's preferred sensory input or motor activity for a task: continued use of paper provided something to 'hear' (hearing the paper drop into the basket); something easy to manipulate (hand held notes); and something to 'deliver' [80,81]. Seniority [42,53], maturity [51] and intention to turnover [60] were linked with workaround behaviours. Psychological gratification and a heroic attitude about their ability and competence to creatively and persistently solve problems and care for their patients without having to depend on a colleague's help, causes many nurses to workaround rather than employ second order problem solving [24]. Laziness offered by a participant is reported in one study as a contributor to circumventing a protocol [41]. However, evidence from the reviewed studies suggests that workaround behaviours reflect nurses' attempts to deliver patient centred care when workflow processes make that difficult $[48,49]$ and that they are more likely to bend the rules if distressed and when morale is low [43]. In their study examining nurses' use of first order problem solving Tucker and Edmondson (2003) draw on observational data to specify that it is "not because nurses are uncommitted, lazy, or incompetent" [59:63]. Nurses are more likely to engage in second order problem solving (less likely to rely on workarounds) when they are motivated and feel psychologically safe to do so [58].

\section{Social and professional factors}

Evidence offered by some studies suggests that workaround behaviours are influenced by relational factors. To illustrate, evaluation of the impact of CPOE on nurse-physician communication identified that whether or not nurses informally acted on verbal orders before they were entered in the CPOE was dependent on their professional relationship and trust in the physician [49]. Workarounds, described as 'situated' practices [48,56], are enabled by collaboration and a belief that the rules are negotiable $[42,56,66]$.

Workarounds were used because of poor communication or to enhance communication and coordination of interrelated tasks between co-working professionals $[48,72,78,81,83]$, to avoid possible or actual inter professional confrontation $[44,58,59,61]$, or because of inter professional etiquette $[52,66]$ or lack thereof (e.g. nurses being logged out of BCMA while they are still using it [84] or ignoring nurses' input about a patient's care [24]). An emphasis on individual vigilance and a professional expectation that nurses will solve problems contributed to workarounds being implemented [44,58,59]. This notion is captured in the words of a nurse interviewed in one of the reviewed studies, "working around problems is just part of my job" [59:61].

\section{Proliferation of workarounds}

There was evidence from the reviewed studies that collaboration enables workarounds to continue and proliferate $[42,49,54,56,66,74,81]$. Enactment of workarounds relies on willingness of others to help. Kobayashi et al. (2005) indicated that a "workaround cannot be effective if the persons involved are not able or willing to perform. Initiators of workarounds take their tacit knowledge of others' skills and abilities into account when deciding how to implement workarounds" [74:1563]. Workarounds are shared or passed on informally [40,53, $54,59,69,71,86$ ] particularly from senior to junior staff, they are observed and absorbed by other professionals and become part of the group behaviour [62].

Workarounds persist because of an emphasis on efficiency $[59,62,72]$, an expectation that staff will solve 
problems $[24,44,58,59,72]$, the autonomy of clinicians $[56,62]$ and lack of role clarity [52]. The ambiguous nature of operational failures and the expectation that they are part of work routine [1] and the diverse relationships between causes and workarounds also contribute to their persistence [4]. When facing workflow blocks, rather than necessarily asking those best equipped to correct problems, nurses ask those who are socially close so as to protect their reputation of competence, thus perpetuating workarounds rather than engaging in second order problem solving [59]. Workarounds proliferate when human resource management activities reinforce them [60], in a culture and climate that supports unsafe practices $[40,41,59]$, rather than reporting of them [87]. Conversely, an organisational culture that promotes psychological safety [58,59], executive dedication [85], supportive leadership and assistance with root cause problem solving [58,59,82,85], compliance checking [85], simplifying processes and decreasing ambiguity [62] will slow the propagation of workarounds.

\section{The perceived impact of workarounds}

While it was implicit that workarounds circumvent workflow blocks and ergo deliver care, we examined papers for explicit perceptions of the impact of workarounds. A small number of studies reported the impact of the workaround practices in terms of measured outcomes, including the estimated cost in nursing time spent on workarounds [59] and the impact of safety workarounds on occupational injuries [79]. In relation to patient safety, not checking patient identification was found to be significantly associated with making an intravenous medication administration error [46]. There were no studies that measured the positive impact of workarounds for patient safety although these were suggested by some studies [e.g. 36, 78]. For the most part, studies propose potential effects of workarounds rather than provide empirical evidence for their impact. Studies were examined for evidence of potential effects of workarounds. These are grouped according to their perceived negative or positive impact in relation to patients, staff and the organisation (Table 5). Several studies identified that workarounds could be both positive and negative $[1,24,48,58,59,71,82]$ depending on the context [69] and the expertise of those using the workarounds [54]. More studies highlighted a negative [4,6,34,40,43, $45,46,49,51,61-64,68-70,83-87]$ rather than positive $[42,53,60,66,67,81]$ impact of workarounds.

\section{Nurses' conceptualisation and rationalisation of workarounds}

Less than a third of the reviewed studies explicitly examine nurses' conceptualisations or rationalisation of their own and their colleagues' workaround behaviours (including rule subversion, first order problem solving, deviations, violations, error re-definition) $[1,36-39,42,44$, $52,58,59,64,66,69,71,82,87]$. Mostly conclusions in relation to this issue are not explicit. Tension in the way workarounds are perceived by nurses emerged in the evaluation of studies. On the one hand, studies reported workaround behaviours as necessary to deliver care or in the best interest of the patient $[1,6,36,42,44,56,59$, $66,67,69,71,72,75,80,81,84,86]$. However, nurses also identified them as unsafe in particular contexts $[69,87]$ and as workarounds are not legally sanctioned, some nurses perceived them as professionally risky $[36,44$, 52,66].

Workarounds were justified through autonomy of practice [62] and rationalised in some studies as acceptable when deemed not to jeopardise patient safety $[40,69,87]$, in emergency situations $[4,42,44,67,71]$, when the nurse is familiar with the patient $[6,41,45]$, when the doctors' response is predictable [66] and when the behaviours fall within the scope of the nurse's knowledge and skill $[44,66]$. However, nurses also reported that not adhering to policy undermined professional ideals and quality of care $[38,87]$ and some workarounds were considered malpractice by nursing leaders [82].

A contradiction in the perceived relationship between workaround behaviours and competency was also evident in a few studies. Fixing problems and working around rules for the sake of the patient were linked with perceived proficiency and satisfaction $[59,66]$ and "the ability to circumvent problems validated nurses' confidence in their competence and professionalism" [24:129]. Rules were perceived as flexible and while on the one hand part of being a 'good nurse' was the ability to use one's judgement to workaround the rules for the benefit of the patient, to do so risked colleagues' perceptions that one was not a 'good nurse' [66]. As workaround behaviours are not legally sanctioned, they can be viewed poorly by colleagues [36,38] and not accommodated for by 'mediocre' [66] and casual or non permanent nurses [42]. Expertise and patient criticality influenced the number and type of deviations from standard protocols in a critical care environment [73].

One study provides evidence that nurses perceive workarounds and breaking protocol, both terms for violations, as different concepts. This study, investigating violations in medication administration, found that working around and breaking protocol "did not fit together as a measure, and the lack of overlap between the predictors of working around protocol and breaking protocol offer evidence that the two terms measure different concepts" [65:748]. That violations and improvisations are understood to mean different things is highlighted by the findings of two studies examining attitudes to patient care behaviours that comply, violate or 
Table 5 The potential effects of workarounds in acute care settings for patients, staff and organisation

\begin{tabular}{|c|c|c|c|}
\hline & Patient & Staff & Organisation \\
\hline \multirow[t]{4}{*}{$\begin{array}{l}\text { Positive } \\
\text { effects }\end{array}$} & \multirow{2}{*}{$\begin{array}{l}\text { - Care is delivered according to the patient's } \\
\text { specific needs [42,67]. For example, } \\
\text { 'batching' care so that the patient can get a } \\
\text { good night sleep; giving medications early } \\
\text { so that they won't be four hours late [42] }\end{array}$} & $\begin{array}{l}\text { - Decrease stress for manager and } \\
\text { other staff [42] }\end{array}$ & $\begin{array}{l}\text {-Workarounds may lead to better } \\
\text { rules [66] }\end{array}$ \\
\hline & & \multirow[t]{3}{*}{ • Increase efficiency and support work [76] } & \multirow[t]{3}{*}{$\begin{array}{l}\text { - Provide excellent information } \\
\text { for improvement efforts }[81,82]\end{array}$} \\
\hline & - Circumvent barriers to delivering care $[56,67]$ & & \\
\hline & $\begin{array}{l}\text { - Annotating printed paper patient } \\
\text { information sheets rather than only } \\
\text { viewing information in EHR, enables } \\
\text { clinicians to acquaint themselves more } \\
\text { with the patients [53] }\end{array}$ & & \\
\hline \multirow[t]{8}{*}{$\begin{array}{l}\text { Negative } \\
\text { effects }\end{array}$} & \multirow{2}{*}{$\begin{array}{l}\text { - Decrease patient safety by increasing the } \\
\text { potential for error } \\
{[4,6,34,40,41,43,45-49,51,61-64,68-70,82-87]}\end{array}$} & $\begin{array}{l}\text { - Make staff vulnerable to retribution } \\
{[37,39,44,66,67]}\end{array}$ & \multirow{2}{*}{$\begin{array}{l}\text { - Prevent organisational learning and } \\
\text { improvement through hiding problems } \\
\text { and practices that are occurring in real } \\
\text { time }[1,6,24,47,56,58,59,72]\end{array}$} \\
\hline & & \multirow{2}{*}{$\begin{array}{l}\text { - Time consuming, erode staff time and } \\
\text { energy or increase cognitive effort } \\
{[48,49,58,59,72,74,82]}\end{array}$} & \\
\hline & \multirow{3}{*}{$\begin{array}{l}\text { - Do not accurately reflect patient care } \\
\text { delivery (e.g. charting a medication earlier } \\
\text { than it was given) }[6,48,61,84] \\
\text { - Decrease surveillance of patients [72] }\end{array}$} & & \multirow{2}{*}{$\begin{array}{l}\text { - Create problems elsewhere in the system } \\
\text { and can lead to other workarounds } \\
{[4,24,48,59,62,74]}\end{array}$} \\
\hline & & \multirow{3}{*}{$\begin{array}{l}\text { - Increase the risk of occupational injuries [79] } \\
\text { - Informal teaching of workarounds is } \\
\text { problematic because there is no clarity } \\
\text { about what clinicians are being taught [53] }\end{array}$} & \\
\hline & & & - Directly or indirectly cost hospitals money \\
\hline & equipment [72] & & {$[1,24,59]$} \\
\hline & $\begin{array}{l}\text { - Loss of information about patients } \\
{[49,71,75,76,81]}\end{array}$ & \multirow[t]{2}{*}{$\begin{array}{l}\text { - Enable staff to express emotion to } \\
\text { coordinate and work more effectively [75] }\end{array}$} & $\begin{array}{l}\text { - Contribute to a culture of unsafe } \\
\text { practices }[40,62]\end{array}$ \\
\hline & - Create new pathways to error [81] & & $\begin{array}{l}\text { - Potentiate security breaches (e.g. nurses } \\
\text { borrowing access codes and posting } \\
\text { them for easy viewing) [69] }\end{array}$ \\
\hline \multirow{7}{*}{$\begin{array}{l}\text { Both } \\
\text { positive } \\
\text { and } \\
\text { negative } \\
\text { effects }\end{array}$} & \multirow{2}{*}{$\begin{array}{l}\text { - In some instances workarounds enhance } \\
\text { patient care but they can also potentiate } \\
\text { patient harm }[4,24,48,69,71]\end{array}$} & $\begin{array}{l}\text {-Workarounds may ease and accelerate } \\
\text { performance but increase workload [48] }\end{array}$ & \multirow{3}{*}{$\begin{array}{l}\text { - Allow the use of CPOE but hide } \\
\text { opportunities for redesign and } \\
\text { improvement [47] } \\
\text { - Allow the system to continue functioning } \\
\text { but may lead to widespread instability } \\
\text { [74] }\end{array}$} \\
\hline & & \multirow{2}{*}{$\begin{array}{l}\text { - Help with the coordination of work and } \\
\text { reduce cognitive load by providing } \\
\text { solutions to recurring problems but lead to } \\
\text { unstable, unavailable or unreliable work } \\
\text { protocols [74] }\end{array}$} & \\
\hline & $\begin{array}{l}\text { - Workarounds fix problems so that patient } \\
\text { care can continue but in not addressing the } \\
\text { underlying problem similar problems may } \\
\text { reoccur in relation to patient care }[1,58,59]\end{array}$ & & \\
\hline & \multirow{2}{*}{$\begin{array}{l}\text { - While one workaround may prevent } \\
\text { medication errors (e.g. using a STOP stamp } \\
\text { on the paper medication chart to indicate } \\
\text { that a medication has been ceased } \\
\text { because the stop and the start orders in } \\
\text { the CPOE look very similar) other } \\
\text { workarounds using the same system } \\
\text { increase error risk (e.g. recording actual } \\
\text { administration times on paper medication } \\
\text { chart but not in the CPOE) [48-50] }\end{array}$} & $\begin{array}{l}\text { - Fix problems so that patient care can } \\
\text { continue but in not addressing the } \\
\text { underlying problem similar problems will } \\
\text { occur requiring staff to address them again } \\
{[58,59]}\end{array}$ & \\
\hline & & \multirow[t]{3}{*}{$\begin{array}{l}\text { - Workarounds may circumvent problematic } \\
\text { EPR-mediated communication between } \\
\text { staff but may also create confusion if the } \\
\text { workaround is not explained [54] }\end{array}$} & \\
\hline & $\begin{array}{l}\text { - Informal handover of information to } \\
\text { workaround the lack of formal } \\
\text { communication channels reduced falls but } \\
\text { may create gaps in passed on patient } \\
\text { information [78] }\end{array}$ & & \\
\hline & $\begin{array}{l}\text { - Deviations are linked with good patient } \\
\text { outcomes (innovations) and bad patient } \\
\text { outcomes (errors) [73] }\end{array}$ & & \\
\hline
\end{tabular}

Legend: EHR (Electronic Health Record); CPOE (Computer Physician Order Entry).

improvise in relation to protocols. These report that while healthcare workers and the public view violations as inappropriate, the opposite is true for compliance regardless of patient outcome. Attitudes to improvisations were influenced by outcome for the patient $[37,39]$. Thus nurses perceived that improvisations were acceptable if the outcome for the patient was good. Violations on the other hand were viewed as inappropriate regardless of outcome [37,39].

\section{Discussion}

Our findings build on and extend the work of Halbesleben et al (2008) [16] and Alper and Karsh (2009) [3]. Although the literature examining nurses' use of workarounds has increased since 2008, there are still relatively few peer reviewed studies examining nurses' workaround behaviours as a primary focus and most that do are located in the USA. There is considerable heterogeneity in the aim, methods, settings and focus of 
the reviewed studies. Some studies observe the frequency and causes of workarounds; others examine attitudes of professionals to circumvention of rules. There are few studies that examine the effect of workaround behaviours in terms of measured outcomes [16]. Workaround behaviours, for example, have been shown to consume organisational resources [59], impact on health professionals occupational health and safety [79] and patient medication safety [46]. However, for the most part, the consequences of workarounds are offered tentatively rather than being solely empirically based [16]. Workarounds have a cascading effect often impacting other microsystems $[48,74]$ thus their effect may not be immediately evident making it difficult to harness and quantify their impact.

Contributing to the relatively underdeveloped body of healthcare research focused on workarounds, given their influence on patient safety, is the difficulty in investigating them. This underlies the use of multiple rather than single research approaches to uncover workarounds' interwoven processes and characteristics [4]. While survey questionnaires have been employed [37,39,43,44,55, 60,63-65,79,83,87], the primary methods used in the reviewed studies included a combination of observation and interviews $[1,6,34,40,41,45,53,54,56,58,59,61,66$, 68,72,74-76,82,84], which are resource intensive. In addition, the possibility for such research to identify glitches or deficiencies in technology and workers 'breaking' rules is fraught with potential implications, that is, financial, legal and political [88].

Workarounds both straddle and widen the gaps in health care delivery [89]. Overall they are reported negatively. There are claims that their implementation: destabilises patient safety $[4,49,61,63,77]$; undermines standardisation [56,62]; increases physical and cognitive workload $[49,59,72,82]$; hides actual practice and opportunities for improvement thus preventing organisational learning $[1,6,24,58,59,84,86]$; and creates further problems and workarounds [24,48,56,59,72,74]. However, other accounts of workarounds describe them as mindful behaviours [60] that provide opportunities for improvement [48] and both compromise and promote patient safety $[48,53]$. Nurses justify workarounds as necessary circumventions to deliver timely and customised patient-centred care in complex and highly variable systems $[36,42,44,47,48,56,58,61,66,67,69,76,80,81,84]$. The potential pathways of workarounds to innovation and excellence and the connection of workarounds with resilience are being recognised [26-28,90].

Studies demonstrate that workarounds are individually or collectively enacted. When enacted as a collective process, they rely heavily on: a shared view that rules are flexible $[42,56,66]$; a tacit agreement to enact $[42,44,52$, $56,66]$; and an understanding of who will and will not workaround [74]. There is some evidence, from a small number of studies, that group norms $[40,42,58,59,86]$, local and organisational leadership $[58,59,82,85]$, professional structures $[24,59,74]$ and relationships [49] and others' expectations $[44,56,58,59,66,74]$ influence the implementation of workarounds. Despite the collegial nature of nursing work and the demonstrated effect of organisational and local culture on clinicians' behaviour and attitudes [91,92], the influence of social networks, relationships, expectations and local and organisational culture on the enactment and proliferation of workarounds is under investigated.

There are suggestions that nurses' notions of what constitutes a 'good' nurse, their ideologies, knowledge and experience, influence their implementation of workarounds [24,59,66]. For example, nurses viewed problem solving as part of nursing and perceived that an ability to do so alone demonstrated competency. They reported a sense of gratification at being able to solve problems individually, protect patients and deliver care $[24,59]$. There is evidence that nurses justify working around rules and policies for the benefit of the patient $[36,42,66]$. However, the importance of adhering to protocols was considered by other nurses to be central to a professional approach to patient care [38]. Introducing technology incites ambiguity in practice and changes the meaning of nursing work [93] which may undermine confidence and threaten a professional's image.

Workarounds continue to be ill defined [16] with less than half of the studies reviewed offering a definition for workarounds or related concepts. Those that did were primarily published since Halbesleben and colleagues' articulation of this shortcoming in 2008 [16]. The lack of clarity may reflect the uncertainty about how workarounds are conceptualised in clinical settings and by researchers. For example, some authors suggest that workarounds lead to potential errors [34], while others propose that these behaviours are the error [52,83]. Importantly, there is lack of clarity in how nurses themselves differentiate workarounds from related constructs [65]. Contributing to the confusion is that some workarounds are viewed as normal practice, with clinicians being unaware that they are in fact workarounds. Furthermore, at times informal workarounds become sanctioned practices [48]. Imprecision in how workarounds are defined and reported poses challenges for researchers and those who would synthesise the evidence.

This scoping review identifies gaps in the literature, which offer opportunities for future research. Further studies are needed that investigate nurses': workarounds as a primary focus; individual and collective conceptualisation of their own and their colleagues workarounds in situ; workaround behaviours and measured patient outcomes; team and organisational cultures on the enactment and proliferation of workarounds. 


\section{Limitations}

This review examined empirical peer reviewed studies written in English. A limitation of literature reviews is that imposed by research and publication timelines, which create a lag between those studies included in the review and new published information. While every attempt was made to capture all published papers in this area using systematic and comprehensive search strategies, some may have been missed.

The main challenge in studies of this type is that workaround behaviours are difficult to delineate from other behaviours [16]. We applied an operational definition of workarounds to behaviours described in the reviewed studies and were inclusive rather than exclusive. It is possible that we missed some workaround behaviours. Alternatively it is possible that we included some behaviours that may not be workaround behaviours. We attempted to ameliorate this effect by employing two reviewers to independently cross-examine randomly selected studies in phases one and two and all of the studies in phase three.

\section{Conclusion}

Workarounds operate as a dichotomous trope. They enable yet potentially compromise patient care and safety. They provide and hide information about clinicians' work. They are individually and collectively enacted. Organisational, work process, patient-related, individual, social, and professional factors, group norms, local and organisational culture, image management and collegiality influence the development, implementation and maintenance of workarounds. As nurses comprise the majority of the healthcare workforce, it is important to understand the use of workarounds in this population. Understanding nurses' practice and their perception of workaround behaviours is at the heart of apprehending how to improve healthcare at the bedside, where care is delivered.

\section{Competing interests}

The authors declare that they have no competing interests.

\section{Authors' contributions}

DD participated in the design of the study, carried out the literature search and selection process, analysed and synthesised the literature and drafted the paper. DG also participated in the design of the study, the literature selection process and helped to analyse, synthesise and draft the paper. JL helped to analyse, synthesise and draft the paper. JB, DB, JT and JJ helped to design the study and to draft the paper. All the authors read and approved the final manuscript.

\section{Acknowledgements}

We would like to thank the NH\&MRC Patient Safety Program Grant (App I.D.568612/Patient Safety: Enabling and Supporting Change for a Safer and More Effective Health System). We would also like to thank: Monica O'Brien, Research Librarian, University of New South Wales, Sydney, Australia; the peer reviewers of the paper; and the authors of the studies we reviewed.

\section{Author details}

${ }^{1}$ Centre for Clinical Governance Research, Australian Institute of Health Innovation, University of New South Wales, Sydney, NSW 2052, Australia. ${ }^{2}$ School of Public Health and Community Medicine and Centre for Clinical Governance Research, Australian Institute of Health Innovation, University of New South Wales, Sydney, NSW 2052, Australia. ${ }^{3}$ Faculty of Health Sciences, University of Sydney, Sydney, NSW 2141, Australia.

Received: 12 December 2012 Accepted: 7 May 2013

Published: 11 May 2013

\section{References}

1. Tucker AL: The impact of operational failures on hospital nurses and their patients. J Oper Manag 2004, 22(2):151-169.

2. Adobe: Workaround available for vulnerability in versions 8.1 and earlier of Adobe Reader and Acrobat. http://www.adobe.com/support/security/ advisories/apsa07-04.html.

3. Alper SJ, Karsh B-T: A systematic review of safety violations in industry. Accid Anal Prev 2009, 41:739-754.

4. Koppel R, Wetterneck T, Telles JL, Karsh B-T: Workarounds to barcode medication administration systems: their occurrences, causes, and threats to patient safety. J Am Med Inform Assoc 2008, 15(4):408-423.

5. Carayon P: Handbook of Human Factors and Ergonomics in Health Care and Patient Safety. Mahwah: Lawrence Erlbaum Associates; 2007.

6. Patterson ES, Rogers ML, Chapman RJ, Render ML: Compliance with intended use of bar code medication administration in acute and longterm care: an observational study. Hum Factors 2006, 48:15-22.

7. Handel MJ, Poltrock S: Working around official applications: experiences from a large engineering project. In ACM 2011 Conference on Computer Supported Cooperative Work. Hangzhou, China: Association for Computing Machinery; 2011:309-312

8. Gasser $L$ : The integration of computing and routine work. ACM Transactions on Information Systems (TOIS) 1986, 4(3):205-225.

9. Hakimzada AF, Green RA, Sayan OR, Zhang J, Patel VL: The nature and occurrence of registration errors in the emergency department. Int J Med Inf 2008, 77:169-175.

10. Atkinson C, Kuhne T: Reducing accidental complexity in domain models. Softw Syst Model 2008, 7:345-359.

11. Tucker AL, Spear SJ: Operational failures and interruptions in hospital nursing. Health Serv Res 2006, 41:643-662.

12. Strong D, Volkoff $O$, Elmes M: ERP systems, task structure, and workarounds in organizations. In Seventh Americas Conference on Information Systems (AMCIS). Boston: Association for Information Systems; 2001:1049-1051.

13. Boudreau M-C, Robey D: Enacting integrated information technology: a human agency perspective. Organ Sci 2005, 16(1):3-18.

14. Day DL: User responses to constraints in computerized design tools. ACM SIGSOFT Software Engineering Notes 1996, 21(5):47-50.

15. Ferneley E, Sobreperez P: Resist, comply or workaround?An examination of different facets of user engagement with information systems. Eur J Inform Syst 2006, 15(4):345-356.

16. Halbesleben JR, Wakefield DS, Wakefield BJ: Work-arounds in health care settings: literature review and research agenda. Health Care Manage Rev 2008, 33(1):2-12.

17. Bradley AJ, Nurre GS, Ochs W, Ryan J, Dougherty H, Bennett NR, Abramowicz-Reed L, Andersen GC, Crabb WG: Post-launch experience of the Hubble Space Telescope: reflections upon the design and operation. In Space Astronomical Telescopes and Instruments II, Volume 1945. Orlando, FL, United States: Publ by Society of Photo-Optical Instrumentation Engineers; 1993:42-54

18. Hollman WJM: The quantification of workarounds and ways to utilize these ramifications. Massachusetts: Massachusetts Institute of Technology; 2011.

19. Gaba DM: Structural and organizational issues in patient safety: a comparison of healthcare to other high-hazard industries. Calif Manage Rev 2000, 43(1):83-102.

20. Vincent C: Patient Safety. Chichester: Wiley-Blackwell Publishing; 2010.

21. Morath J, Turnbull J: To Do No Harm. San Francisco: Jossey-Bass A Wiley Imprint; 2005.

22. Vassilakopoulou P, Tsagkas V, Marmaras N: Workaround identification as an instrument for work analysis and design: a case study on ePrescription. Work: A J Prevention, Assessment and Rehabilitation 2012, 41(1):1805-1810. 
23. Reason J: The Human Contribution: Unsafe Acts, Accidents and Heroic Recoveries. Surrey: Ashgate Publishing Limited; 2008.

24. Tucker AL, Edmondson A, Spear S: When problem solving prevents organizational learning. J Organisational Change Manage 2002, 15(2):122-136.

25. Whooley O: Diagnostic ambivalence: psychiatric workarounds and the diagnostic and statistical manual of mental disorders. Sociol Health IIIn 2010, 32(3):452-469.

26. Woods D: Essential characteristics of resilience. In Resilience Engineering Concepts and Precepts. Aldershot: Ashgate Publishing Limited; 2006:21-34.

27. Hollnagel E, Woods D, Leveson N: Resilience Engineering Concepts and Precepts. Aldershot: Ashgate Publishing Limited; 2006.

28. Lalley C, Malloch K: Workarounds: the hidden pathway to excellence. Nurse Leader 2010, 8(4):29-32

29. Mays N, Roberts E, Popay J: Synthesising research evidence. In Studying the Organisation and Delivery of Health Services: Research Methods. Edited by Fulop N, Clarke A, Black N. London: Routledge; 2001.

30. Victoor A, Delnoij D, Friele R, Rademakers J: Determinants of patient choice of healtcare providers: a scoping review. BMC Health Serv Res 2012, $12: 272$.

31. Levac D, Colquhoun H, O'Brien K: Scoping studies: advancing the methodology. Implement Sci 2010, 5(1):1-9.

32. Brown KF, Long SJ, Athanasiou T, Vincent CA, Kroll JS, Sevdalis N: Reviewing methodologically disparate data: a practical guide for the patient safety research field. J Eval Clin Pract 2010, 18(1):172-181.

33. Brien S, Lorenzetti D, Lewis S, Kennedy J, Ghali W: Overview of a formal scoping review on health system report cards. Implement Sci 2010, 15(5):12.

34. Ali $M$, Cornford T, Klecun E: Exploring control in health information systems implementation. Stud Health Technol Inform 2010, 160:681-685.

35. Conroy S, Appleby K, Rostock D, Unsworth V, Cousins D: Medication errors in a children's hospital. Paediatric and Perinatal Drug Therapy 2007, 8(1):18-25.

36. Furber C, Thomson A: 'Breaking the rules' in baby-feeding practice in the UK: deviance and good practice? Midwifery 2006, 22(4):365-376.

37. Lawton R, Parker D: Judgments of the rule-related behaviour of health care professionals: an experimental study. Br J Health Psychol 2002, 7(3):253-265.

38. McDonald R, Waring J, Harrison S, Walshe K, Boaden R: Rules and guidelines in clinical practice: a qualitative study in operating theatres of doctors' and nurses' views. Qual Saf Health Care 2005, 14(4):290-294.

39. Parker $D$, Lawton R: Judging the use of clinical protocols by fellow professionals. Soc Sci Med 2000, 51(5):669-677.

40. Taxis $K$, Barber N: Causes of intravenous medication errors: an ethnographic study. Qual Saf Health Care 2003, 12(5):343-347.

41. Dougherty L, Sque M, Crouch R: Decision-making processes used by nurses during intravenous drug preparation and administraton. 2011:1302-1311. Published on line 17

42. Baker HM: Rules outside the rules for administration of medication: a study in New South Wales. Australia. J Nurs Scholarsh 1997, 29(2):155-158.

43. Fogarty GJ, McKeon CM: Patient safety during medication administration: the influence of organizational and individual variables on unsafe work practices and medication errors. Ergon 2006, 49(5-6):444-456.

44. McKeon CM, Fogarty GJ, Hegney DG, McKeon CM, Fogarty GJ, Hegney DG: Organizational factors: impact on administration violations in rural nursing. J Adv Nurs 2006, 55(1):115-123.

45. Popescu A, Currey J, Botti M: Multifactorial influences on and deviations from medication administration safety and quality in the acute medical/ surgical context. Worldviews Evid Based Nurs 2011, 8(1):15-24

46. Westbrook Jl, Rob MI, Woods A, Parry D: Errors in the administration of intravenous medications in hospital and the role of correct procedures and nurse experience. BMJ Quality \& Safety 2011, 20(12):1027-1034

47. Niazkhani Z: Evaluating the impact of CPOE systems on medical workflow: a mixed method study. Stud Health Technol Inform 2008, 136:881-882.

48. Niazkhani Z, Pirnejad H, van der Sijs $H$, Aarts J: Evaluating the medication process in the context of CPOE use: the significance of working around the system. Int J Med Inf 2011, 80:490-506.

49. Pirnejad $H$, Niazkhani $Z$, van der Sijs H, Berg M, Bal R: Evaluation of the impact of a CPOE system on nurse-physician communication - a mixed method study. Methods Inf Med 2009, 48(4):350-360.

50. Van Der Sijs H, Rootjes I, Aarts J: The shift in workarounds upon implementation of computerized physician order entry. Stud Health Technol Inform 2011, 169:290-294.
51. van Onzenoort HA, van de Plas A, Kessels AG, Veldhorst-Janssen NM, van der Kuy P-HM, Neef C: Factors influencing bar-code verification by nurses during medication administration in a Dutch hospital. Am J Health Syst Pharm 2008, 65(7):644-648.

52. Espin S, Lingard L, Baker GR, Regehr G: Persistence of unsafe practice in everyday work: an exploration of organizational and psychological factors constraining safety in the operating room. Qual Saf Health Care 2006, 15(3):165-170.

53. Varpio $L$, Schryer $C F$, Lehoux $P$, Lingard L: Working off the record: physicians' and nurses' transformations of electronic patient recordbased patient information. Acad Med 2006, 81(10):S35-S39.

54. Varpio L, Schryer CF, Lingard L: Routine and adaptive expert strategies for resolving ICT mediated communication problems in the team setting. Med Educ 2009, 43(7):680-687.

55. Otsuka Y, Misawa R, Noguchi H, Yamaguchi $H$ : A consideration for using workers' heuristics to improve safety rules based on relationships between creative mental sets and rule-violating actions. Saf Sci 2010, 48(7):878-884.

56. Azad B, King N: Enacting computer workaround practices within a medication dispensing system. Eur J Inform Syst 2008, 17(3):264-278.

57. Picheansathian W: Compliance with universal precautions by emergency room nurses at Maharaj Nakorn Chiang Mai Hospital. J Med Assoc Thai 1995, 78(Suppl 2):S118-S122.

58. Tucker AL, Edmondson A: Managing routine exceptions: a model of nurse problem solving behavior. Advances in Health Care Management 2002, 3:87-113.

59. Tucker AL, Edmondson A: Why hospitals don't learn from failures: organizational and psychological dynamics that inhibit system change. Calif Manage Rev 2003, 45(1):55-72.

60. Wheeler AR, Halbesleben JRB, Harris KJ: How job-level HRM effectiveness influences employee intent to turnover and workarounds in hospitals. J Bus Res 2012, 65(4):547-554

61. Halbesleben JR, Savage GT, Wakefield DS, Wakefield BJ: Rework and workarounds in nurse medication administration process: implications for work processes and patient safety. Health Care Manage Rev 2010, 35(2):124-133.

62. Mazur LM, Chen S-J: An empirical study for medication delivery improvement based on healthcare professionals' perceptions of medication delivery system. Health Care Manag Sci 2009, 12(1):56-66.

63. Morriss FH Jr, Abramowitz PW, Carmen L, Wallis AB: "Nurses Don't Hate Change" - survey of nurses in a neonatal intensive care unit regarding the implementation, use and effectiveness of a bar code medication administration system. Healthc Q 2009, 12:135-140.

64. Alper S, Holden R, Scanlon M, Patel N, Kaushal R, Skibinski K, Brown R, Karsh B: Self-reported violations during medication administration in two paediatric hospitals. BMJ Qual Saf 2012, 21:408-415.

65. Alper SJ, Holden RJ, Scanlon MC, Kaushal R, Shalaby TM, Karsh BT: Using the technology acceptance model to predict violations in the medication use process. In Proceedings of the Human Factors and Ergonomics Society 51st Annual Meeting, Volume 51. Baltimore, MD, United States: Human Factors and Ergonomics Society; 2007:745-749.

66. Hutchinson SA: Responsible subversion: a study of rule-bending among nurses. Res Theory Nurs Pract 1990, 4(1):3-17.

67. Eisenhauer LA, Hurley AC, Dolan N, Eisenhauer LA, Hurley AC, Dolan N Nurses' reported thinking during medication administration. J Nurs Scholarsh 2007, 39(1):82-87.

68. Carayon P, Wetterneck TB, Hundt AS, Ozkaynak M, DeSilvey J, Ludwig B, Ram P, Rough SS: Evaluation of nurse interaction with bar code medication administration technology in the work environment. J Patient Saf 2007, 3(1):34-42.

69. Zuzelo PR, Gettis C, Hansell AW, Thomas L: Describing the influence of technologies on registered nurses' work. Clin Nurse Spec 2008, 22(3):132-140.

70. Miller DF, Fortier CR, Garrison KL: Bar code medication administration technology: characterization of high-alert medication triggers and clinician workarounds. Ann Pharmacother 2011, 45(2):162-168.

71. Schoville RR: Work-arounds and artifacts during transition to a computer physician order entry: what they are and what they mean. J Nurs Care Qual 2009, 24(4):316-324.

72. Fowler PH, Craig J, Fredendall LD, Damali U: Perioperative workflow: barriers to efficiency, risks, and satisfaction. AORN J 2008, 87(1):187.

73. Kahol K, Vankipuram M, Patel VL, Smith ML: Deviations from protocol in a complex trauma environment: errors or innovations? J Biomed Inform 2011, 44(3):425-431. 
74. Kobayashi M, Fussell SR, Xiao Y, Seagull FJ: Work coordination, workflow, and workarounds in a medical context. In Conference on Human Factors in Computing Systems: CHI '05. Portland, United States: Association for Computing Machinery; 2005:1561-1564.

75. Mentis HM, Reddy M, Rosson MB: Invisible emotion: information and interaction in an emergency room. In 2010 ACM Conference on Computer Supported Cooperative Work. Savannah, GA, United States: Association for Computing Machinery; 2010:311-320.

76. Saleem JJ, Patterson ES, Militello L, Render ML, Orshansky G, Asch SM: Exploring barriers and facilitators to the use of computerized clinical reminders. J Am Med Inform Assoc 2005, 12(4):438-447.

77. Kirkbride G, Vermace B: Smart pumps: implications for nurse leaders. Nurs Adm Q 2011, 35(2):110-118.

78. Lopez KD, Gerling GJ, Cary MP, Kanak MF: Cognitive work analysis to evaluate the problem of patient falls in an inpatient setting. J Am Med Inform Assoc 2010, 17(3):313-321.

79. Halbesleben JR: The role of exhaustion and workarounds in predicting occupational injuries: a cross-lagged panel study of health care professionals. J Occup Health Psychol 2010, 15(1):1-16.

80. Saleem JJ, Russ AL, Justice CF, Hagg H, Ebright PR, Woodbridge PA, Doebbeling BN: Exploring the persistence of paper with the electronic health record. Int J Med Inf 2009, 78:618-628.

81. Saleem JJ, Russ AL, Justice CF, Hagg H, Woodbridge PA, Doebbeling BN: Paper use with the electronic medical record: an important supplement or negative circumvention? In 52nd Human Factors and Ergonomics Society Annual Meeting, Volume 2. New York, NY, United States: Human Factors an Ergonomics Society Inc; 2008:773-777.

82. Zhou X, Ackerman MS, Zheng K: CPOE workarounds, boundary objects, and assemblages. In Conference on Human Factors in Computing Systems: CHI '11. Vancouver, Canada: Association for Computing Machinery; 2011:3353-3362.

83. O'Neil S, Speroni KG, Dugan L, Daniel MG: A 2-tier study of direct care providers assessing the effectiveness of the red rule education project and precipitating factors surrounding red rule violations. Qual Manag Health Care 2010, 19(3):259-264.

84. Patterson E, Cook R, Render M: Improving patient safety by identifying side effects from introducing bar coding in medication administration. J Am Med Inform Assoc 2002, 9:540-553.

85. McNulty J, Donnelly E, lorio K: Methodologies for sustaining barcode medication administration compliance. A multi-disciplinary approach. $J$ Healthc Inf Manag 2009, 23(4):30-33.

86. McAlearney AS, Vrontos J Jr, Schneider PJ, Curran CR, Czerwinski BS, Pedersen CA: Strategic work-arounds to accommodate new technology: the case of smart pumps in hospital care. J Patient Saf 2007, 3(2):75-81

87. Orbe MP, King G 3rd: Negotiating the tension between policy and reality: exploring nurses' communication about organizational wrongdoing. Health Commun 2000, 12(1):41-61.

88. Obradovich J, Woods D: Users as designers: How people cope with poor $\mathrm{HCl}$ design in computer-based medical devices. Hum Factors 1996, 38(4):574-592

89. Debono D, Greenfield D, Black D, Braithwaite J: Achieving and resisting change: workarounds straddling and widening gaps in health care. In The Reform of Health Care. Edited by Dickinson H, Mannion R. London: Palgrave Macmillan; 2012:177-192.

90. Tucker AL: Workarounds and resiliency on the front lines of health care. http://www.webmm.ahrq.gov/perspective.aspx?perspectivelD $=78$.

91. Braithwaite J, Hyde P, Pope C: Culture and Climate in Health Care Organizations. Palgrave Macmillan: Great Britain; 2010.

92. Callen $J \mathrm{~L}$, Braithwaite J, Westbrook Jl: Cultures in hospitals and their influence on attitudes to, and satisfaction with, the use of clinical information systems. Soc Sci Med 2006, 65(3):635-639.

93. Barnard A, Gerber R: Understanding technology in contemporary surgical nursing: a phenomenographic examination. Nurs Inq 1999, 6:157-166.

doi:10.1186/1472-6963-13-175

Cite this article as: Debono et al.: Nurses' workarounds in acute

healthcare settings: a scoping review. BMC Health Services Research 2013 13:175

\section{Submit your next manuscript to BioMed Central and take full advantage of:}

- Convenient online submission

- Thorough peer review

- No space constraints or color figure charges

- Immediate publication on acceptance

- Inclusion in PubMed, CAS, Scopus and Google Scholar

- Research which is freely available for redistribution

Submit your manuscript at www.biomedcentral.com/submit
C BioMed Central 